Research Fund of the University of London for a grant.

\section{U. Ahmad}

21 Carston Close,

London, S.E.12.

Received October 19, revised November 21, 1966

${ }^{1}$ Geikie, A., The Scenery of Scotland, first ed., 17 (Macmillan, London, 1865).

${ }^{2}$ Horne, J., and Hinxman, L. W., Mem. Geol. Surv., 83, 68 (1014).

${ }^{3}$ Bailey, E. B., Mem. Geol. Surv., 53, 215 (1916).

${ }^{4}$ Kennedy, W. Q., Quart. J. Geol. Soc. Lond., 102, 41 (1946).

${ }^{5}$ Shand, S. J., Geol. Mag., 88, 423 (1951). - Collette, B. J., Publicate Mineralogish Institute, Rijks-Unicersitiet Utrecht

7 Flinn, D, Nature, 191, 589 (1961)

${ }^{8}$ Wilson, T. J., Nature. 195, 135 (1962).

${ }^{9}$ Riddihough, R. P., Nature, 203, 747 (1964).

10 Ahmad, M. U., thesis, Eniv. London (1966).

11 Vacquier, V., et al., Geol. Soc. Amer. Mem., 47 (1951).

12 Mould, D. D. C. P., Geol. Mag., 83, 249 (1946).

13 Sabine, P. A., Bull. Geol. Surv. of Great Brit., 20, 1 (1963).

${ }^{14}$ Munro, M., Scot. J. Geol., 1, 152 (1965).

\section{PHYSICS}

\section{Does a Moving Body appear Cool ?}

P. T. LANDSBERG in an interesting note ${ }^{1}$ suggests that the "true" value of temperature of a moving body at relativistic speeds will appear to be the same as the temperature measured in the inertial frame of the body itself. $\mathrm{He}$ reaches this conclusion by re-defining temperature in terms of entropy and of internal energy.

I would like to suggest that the whole concept of the apparent temperature of a moving body needs some physical consideration before any mathematics are employed at all.

Suppose that the moving body consists of a box containing gas at about $0^{\circ} \mathrm{C}$ temperature. Then, if this gas is observed as it moves rapidly past a stationary observer, it will, as is well known, suffer a Fitzgerald-Lorentz contraction in the direction of motion by an amount of $\sqrt{ }\left(1-w^{2} / c^{2}\right)$ or $1 / \beta$, where $w$ is the velocity of the box with respect to the observer. We know, also, that time measured in the box will move more slowly than time as measured by the observer, the same factor being involved.

This means that if we consider any individual molecule in the gas in the box which happens to be moving in the direction of motion of the box, its velocity will appear to our observer to be reduced by a factor $\beta^{2} \times$ the measurement made relative to the box itself, because the distance which the particle moves between two collisions or other events will be reduced and the time between them increased as seen by the observer.

On the other hand, a molecule moving transversely to the box will appear to the observer to cover exactly the same distance relative to the box between two events, in the $\beta \times$ increased time, so that in this case its apparent velocity is reduced only $\beta$-times.

We have thus, in the view of the stationary observer, a set of particles in the box with a distribution of velocity in one direction quite different from that in the two transverse directions, and any arguments about temperature, which is normally taken to refer to isotropic motions at least in a gaseous system, will be very difficult to apply. The mass of each particle will, of course, appear to be increased $\beta$-times to the observer in whatevor direction the particles are moving, but nevertheless the mean relative kinetic energy of the particles will appear to be reduced, even if moving transversely.

On the simplest definition of temperature, therefore, the body will indeod look cooler as a result of its motion, even though the question of how much cooler may be regarded as ambiguous. Temperature could, of course, be defined in a number of ways and doubtloss each of these would give a different answer to the apparent change in relativistic conditions, because, as I have tried to show, the whole idea of temperature must be changed drastically when considering such fast moving bodies.

On the genoral principle that the simplest definition is best, Einstein's original expectation that a moving body would appear to be cool would seem qualitatively valid, while Professor Landsberg's case would seem to be only a selected example of a very much larger class of less simple definitions.

\section{J. H. Fremlin}

Department of Physics,

University of Birmingham,

Edgbaston, Birmingham.

Received December 5, 1966.

${ }^{1}$ Landsberg, P. T., Nature, 212, 571 (1966).

\section{THE SOLID STATE}

\section{Fatigue Crack Propagation in Metals}

A RECENT paper by Pearson ${ }^{1}$ reports the results of crack propagation experiments on various metals, of which Young's moduli $E$ range from $6.5 \times 10^{8} \mathrm{lb}$. $/ \mathrm{in.}^{2}$ to $30 \times 10^{6} \mathrm{lb} . / \mathrm{in} .^{2}$. It was found that the fatigue crack propagation rates were sensibly equal when the applied stress $f$ was a fixed proportion of $E$. I have recently obtained theoretical support for this from the (static) analysis of the stresses in the neighbourhood of a crack in a perfectly elastic sheet. Account is taken of the varying geometry of the crack and it is shown, for example, that near the tip of the crack

$$
\frac{1}{E} \sigma_{y} \sim \frac{1}{2} \ln \left(\frac{2 f^{2} c}{E^{2} x}\right)
$$

where $x$ is measured from the tip and in line with the crack, $2 c$ is the length of the crack, and $\sigma_{y}$ the direct stress normal to the line of the crack. The important points to notice from this equation are that the stresses are not proportional to the applied load and, furthermore, they depend on the value of Young's modulus itself. A measure of the extent of the stress singularity is given by equating to unity the terms in parentheses, whence

$$
\bar{x}, \text { say }=\frac{2 f^{2} c}{E^{2}}
$$

The significance of the parameter $\frac{f}{E} \sqrt{ } c$ follows immediately from the assumption that the crack propagation rate depends primarily on the distance $\bar{x}$.

\section{E. H. Mansfield}

Structures Department,

Royal Aircraft Establishment,

Farnborough, Hampshire.

Received December 19, 1966.

${ }^{1}$ Pearson, S., Nature, 211, 1077 (1966).

\section{Amorphous Nucleation in Metals}

Evidence from metal films grown inside electron diffractometers suggests that the earliest stage of film growth, when metals are deposited on amorphous substrates, is the formation of completely disordered clusters of atoms. In face-centred cubic metals the average thickness for this stage of growth is less than $10 \AA$, but with some body-centred cubic metals ${ }^{1,2}$ the amorphous phase persists to thicknesses more than $50 \AA$. 